\title{
14th Trinational Meeting of the German Surgical Working Group for Minimally Invasive Surgery (CAMIC), the Austrian Working Group for Minimally Invasive Surgery (AMIC) and the Swiss Association for Laparoscopic and Thoracoscopic Surgery (SALTS)
}

\author{
14. Dreiländertreffen der Deutschen Chirurgischen Arbeitsgemeinschaft für Minimal \\ Invasive Chirurgie (CAMIC), der Österreichischen Arbeitsgemeinschaft Minimal Invasive \\ Chirurgie (AMIC) und der Schweizerischen Arbeitsgemeinschaft für Laparo- und \\ Thorakoskopische Chirurgie (SALTC)
}

February 18th-19th 2016, Heidelberg, Germany

\author{
Organizing Committee \\ Prof. Dr. Beat P. Müller-Stich \\ Chirurgische Universitätsklinik Heidelberg \\ Klinik für Allgemein-, Viszeral- und Transplantationschirurgie \\ Im Neuenheimer Feld 110 \\ 69120 Heidelberg \\ Germany \\ Prof. Dr. Kaja Ludwig \\ Klinikum Südstadt Rostock \\ Klinik für Allgemein-, Viszeral-, Thorax- und Gefäßchirurgie \\ Südring 81 \\ 18059 Rostock \\ Germany
}

\section{Congress Venue}

Kongresshaus Stadthalle Heidelberg

Neckarstaden 24

69117 Heidelberg

\section{D.1}

Mobile, real-time and point-of-care augmented reality: a pilot study in a phantom, animal and human model

Hannes Kenngott ${ }^{1}$, Anas Preukschas ${ }^{1}$, Martin Wagner ${ }^{1}$, Felix Nickel ${ }^{1}$, Anna-Laura Wekerle ${ }^{1}$, Nadine Bellemann ${ }^{1}$, Boris Radeleff ${ }^{1}$, HansUlrich Kauczor $^{1}$, Michael Müller ${ }^{2}$, Hans-Peter Meinzer ${ }^{2}$, Matthias Gondan $^{1}$, Christian Stock ${ }^{1}$, Markus Büchler ${ }^{1}$, Beat P. Müller-Stich

${ }^{1}$ Universitätsklinikum Heidelberg, Heidelberg, Germany; ${ }^{2}$ Deutsches Krebsforschungszentrum, Heidelberg, Germany

Background: Medical imaging is essential for the diagnosis and therapy of patients. Imaging data is required at the patient's bedside, but is usually accessed at desktop workstations. Advances in mobile technology makes real-time, point-of-care medical applications possible. Our aim was to evaluate such an augmented reality system for medical diagnosis and therapy with regard to feasibility and accuracy in a pilot study involving phantom, animal, and human models.

Methods: After computed tomography imaging a tablet computer was positioned above the patient and a semi-transparent 3D-representation of structures of interest were superimposed on top of the patient's image. Live camera images and the 3D-Volume were registered by fiducial markers. Feasibility and accuracy were evaluated in a static model using the open source Heidelberg Laparoscopy Phantom. The system was further analyzed in a porcine animal study. Finally the setup was tested with a human volunteer.

Results: In the phantom model of the 1380 analyzed AR-positions $83.9 \%$ could be successfully realized. The reprojection error was $2.83 \pm 2.68 \mathrm{~mm} .95 \%$ of the measurements were below $6.71 \mathrm{~mm}$. In the animal model $79.3 \%$ of the 690 analyzed AR-positions could be successfully realized. In the animal study the reprojection error was $3.52 \pm 3.00 \mathrm{~mm}$. $95 \%$ of the measurements were below $9.49 \mathrm{~mm}$. The reprojection error was significantly lower in the phantom model compared to the porcine model $(P<0.001)$. At last augmented reality was successfully realized in clinical case.

Conclusion: Mobile, real-time and point-of-care augmented reality systems for clinical purposes are feasible and accurate in a realistic experimental setting.

kenni@gmx.de 
3D.2

Predicting good parathyroid function after thyroid surgery using parathyroid angiography with ICG fluorescence

Jordi Vidal Fortuny ${ }^{1}$, Samira Sadowski ${ }^{1}$, Valentina Belfontali ${ }^{1}$, Wolfram Karenovics ${ }^{1}$, Sébastien Guigard ${ }^{1}$, Frédéric Triponez ${ }^{1}$

${ }^{1}$ University Hospitals of Geneva, Switzerland

Background: Post-operative hypoparathyroidism remains the most common complication after thyroidectomy. The aim of this pilot study was to evaluate the use of intra-operative parathyroid gland angiography in predicting the function of each individual parathyroid gland and the absence of post-operative hypoparathyroidism.

Methods: In patients undergoing total thyroidectomy, angiography with the fluorescent dye indocyanine green (ICG) was performed after thyroid resection to visualize the vascularization of the identified parathyroid glands. Results: Thirty-six patients underwent ICG-angiography during thyroidectomy. All patients received standard calcium and Vitamin D supplementation (calcium $1 \mathrm{~g}$ and Vitamin D $400 \mathrm{UI}$ bid). A well-vascularized parathyroid gland was demonstrated by ICG-angiography in 30 patients. In these patients, the PTH and calcium levels on post-operative day (POD) 1 were $3.3 \pm 1.4 \mathrm{pmol} / 1$ and $2.27 \pm 0.1 \mathrm{mmol} / 1$, respectively, and on POD 10, $4.0 \pm 1.6 \mathrm{pmol} / \mathrm{l}$ and $2.32 \pm 0.08 \mathrm{mmol} / 1$, respectively. All 30 patients had PTH levels in the normal range on PODs 1 and 10, and only one patient exhibited asymptomatic hypocalcaemia on POD 1 . Two of the 6 patients in whom no well-vascularized parathyroid gland could be demonstrated developed transient hypoparathyroidism. None of the 36 patients presented symptomatic hypocalcaemia and none of them needed Rocaltrol treatment.

Conclusion: In this pilot study, the PTH levels on POD 1 were normal in all the patients who had at least one well-vascularized parathyroid gland, as demonstrated intra-operatively by ICG-angiography, suggesting that ICG-angiography could be a reliable tool to predict the absence of postoperative hypoparathyroidism after thyroidectomy.

frederic.triponez@hcuge.ch

\section{D.4 \\ ICG-fluorescence angiography demonstrates the good function of parathyroid remnant during subtotal parathyroid}

Jordi Vidal Fortuny ${ }^{1}$, Samira Sadowski ${ }^{1}$, Valentina Belfontali ${ }^{1}$, Sébastien Guigard $^{1}$, Wolfram Karenovics ${ }^{1}$, Frédéric Triponez ${ }^{1}$

${ }^{1}$ University Hospitals of Geneva, Switzerland

Background: The 2 major complications of subtotal parathyroidectomy are persistent hyperparathyroidism (HTP) and definitive hypoparathyroidism. We report our first 9 patients in which the vascularization of the parathyroid remnant was verified by intraoperative parathyroid angiography before resecting the 3 other enlarged glands.

Methods: Between May 2014 and May 2015, 64 patients underwent parathyroidectomy in our center including 9 who underwent subtotal parathyroidectomy with intraoperative angiography using the fluorescent dye IndoCyanine Green (ICG) (6 patients with renal HPT including one with simultaneous total thyroidectomy, one patient with Lithium-induced primary HPT, one patient with MEN1a associated primary HPT and one patient with 4 glands hyperplasia). Calcium and parathormone levels were measured at day 1,10 and $3.7 \pm 1.6$ month postoperatively. All patients received systematic Calcium $(1 \mathrm{~g}$ tid) and $1.25-\mathrm{OH}-$ Vitamin D (1 ug bid) supplementation.

Results: ICG angiography showed a well-vascularized remnant in all patients. At follow up, PTH levels dropped $76 \pm 6.5 \%$ from the preoperative levels and all patients had calcium $(2.07-2.36 \mathrm{mmol} / \mathrm{L})$ and PTH levels in the normal range (3.0-13.6 pmol/L).
Conclusion: Intraoperative ICG angiography demonstrated the good perfusion and function of the parathyroid remnant following subtotal parathyroidectomy. It is currently the only available tool assessing the function of parathyroid remnant intraoperatively.

frederic.triponez@hcuge.ch

\section{D.5}

Near-Infrared fluorescence is useful for identification of the intersegmental plane in VATS lung segmentectomy

Sébastien Guigard ${ }^{1}$, Jordi Vidal Fortuny ${ }^{1}$, Marc Licker ${ }^{1}$, Frédéric Triponez ${ }^{1}$, Wolfram Karenovics ${ }^{1}$

${ }^{1}$ University Hospitals of Geneva, Switzerland

Background: Evaluation of fluorescence imaging during lung segmentectomy by Video-Assisted Thoracic Surgery.

Methods: Study of VATS segmentectomies assisted by fluorescence imaging (PINPOINT ${ }^{\circledR}$ Novadaq, Canada) in the University Hospitals of Geneva from November 2014 to August 2015. Analysis of technical input, pathological findings, postoperative course and cTNM / pTNM correlation.

Results: Intersegmental plane identification was perfect in all 17 consecutive segmentectomies, providing additional assistance in case of difficulty with anatomical vascular segmentation. One patient was converted to lobectomy by thoracotomy because of oncological reasons. The postoperative course was uneventful except in 1 patient who developed pneumonia and prolonged air leak (10 days). The drain was removed on POD1 or POD2 in 16 patients with a mean hospital stay of $4.9+/-2.3$ days. Resections were complete for 4 benign lesions and 13 lung cancers.

Conclusion: Fluorescence imaging provides a technical assistance for intersegmental plane identification in VATS, and facilitates vascular identification. It contributes to the quality of diagnostic and therapeutic excisions of small nodules which are often not visible and not palpable during VATS.

frederic.triponez@hcuge.ch

\section{BD.2}

Laparoscopic repair versus open sublay: the influence of risk factors on the outcome of incisional hernia repair

Thomas Simon ${ }^{1}$, Ferdinand Koeckerling ${ }^{2}$

${ }^{1}$ GRN-Klinik Weinheim, Germany; ${ }^{2}$ Vivantes Klinikum Spandau, Germany

Background: Laparoscopic mesh repair for incisional hernias is increasingly used compared to the open hernia repair with a sublay mesh. The purpose of this study was to investigate the influence of risk factors on the peri- and postoperative outcomes after incisional hernia repair in a large, prospectively collected hernia-specific database.

Methods: The German hernia database (Herniamed) collects prospectively data from over 400 participating centers mainly from Germany, Austria and Switzerland into a central registry. For this study, only patients undergoing an elective primary repair of median incisional hernias in either open sublay or laparoscopic intraperitoneal onlay mesh technique were included. Patients with previous hernia repairs, different kinds of ventral hernias and patients younger than 18 years were excluded. Univariate and multivariate analysis were performed to determine the effects of age, gender, body mass index (BMI), operation method, existence of risk factors, defect size, defect closure, and defect localization on outcomes. Results: From 211,894 patients in the database, 3393 were enrolled in this study. Of these, 1655 patients (48.78\%) underwent laparoscopic hernia mesh repair whereas 1738 patients $(51.22 \%)$ underwent open sublay hernia repair. There was no difference regarding overall intraoperative complications $(2.96 \%$ vs. $2.13 \%, p=0.127)$ but laparoscopic hernia 
repair had a higher rate of intraoperative bleedings ( $1.33 \%$ vs. $0.35 \%$, $p<0.002)$. The rate of general complications (3.08\% vs. $4.89 \%$, $p<0.008)$, operation specific postoperative complications $(5.32 \%$ vs. $12.20 \%, p<0.001)$ and reoperation rate $(2.1 \%$ vs. $4.8 \%, p<0.001)$ were significantly higher for patients after open sublay repair. Laparoscopic repair showed a significantly higher recurrence rate $(6.8 \%$ vs. $3.9 \%, p<0.001)$. Regarding postoperative complications, laparoscopic hernia repair was the strongest, protective factor (OR 0.481 CI $0.367-$ $0.631, p<0.001)$ whereas the laparoscopic approach increased the recurrence rate (OR $1.8171 .314-2.512, p<0.001)$.

Conclusion: Laparoscopic incisional hernia repair protects from postoperative complications and reoperations. However, the better postoperative outcome comes at the prize of a higher rate of intraoperative bleedings and recurrences.

thomas.simon@grn.de

\section{BD.4}

\section{Hernia surgery with long term resorbable mesh}

Andreas Koch ${ }^{1}$

${ }^{1}$ Praxis für Viszeral- und Hernienchirurgie, Cottbus, Germany

Background: Not every hernia requires a permanent synthetic mesh. The ongoing chronic inflammatory reaction could be a reason for chronic pain. A long term resorbable mesh allows a reinforcement without long term complications of synthetic meshes. The combination of a reinforcement with such a long term resorbable mesh based on Monofilament Poly-4-hydroxybuterate ( $\mathrm{P} 4 \mathrm{HB}$ ) which is biologically produced and not chemically synthesized and an anatomic restoration of the inguinal floor allows closing the gap between pure tissue and permanent mesh repairs especially in cases of Type II direct and combined inguinal hernias. The mesh is placed in the prepertioneal space (size $8 \times 12 \mathrm{~cm}$ ) and the inguinal floor is restored by a Shouldice repair.

Methods: The first 20 cases were followed up with the carolina comfort scale. Results: There were no severe complications, no recurrences and no differences in the CCS scores compared to Gilbert and Lichtenstein Repairs after 12 weeks.

Conclusion: The "Reinforced" Shouldice Repair is a excellent choice for repairs without a permanent mesh as a part of an tailored approach. The early experiences shows no difference to the established procedures.

info@chirurgie-cottbus.com

\section{CAS.1}

Robotic camera assistance and its benefit in $\mathbf{1 0 3 3}$ traditional laparoscopic procedures: prospective clinical trial

Sebastian Holländer ${ }^{1}$, Dieter Birk ${ }^{2}$

${ }^{1}$ Universitätsklinikum des Saarlandes, Germany; ${ }^{2}$ Krankenhaus Bietigheim, Germany

Background: Despite advances in instruments and techniques in laparoscopic surgery, one thing remains uncomfortable: the camera assistance. The aim of this study was to investigate the benefit of a joystick-guided camera holder (SoloAssist ${ }^{\circledR}$, Aktormed, Barbing, Germany) for laparoscopic surgery and to compare the robotic assistance to human assistance. Methods: One thousand thirty-three consecutive laparoscopic procedures were performed assisted by the SoloAssist ${ }^{\circledR}$. Failures and aborts were documented and nine surgeons were interviewed by questionnaire regarding their experiences.

Results: In 71 of 1033 procedures, robotic assistance was aborted and the procedure was continued manually, mostly because of frequent changes of position, narrow spaces, and adverse angular degrees. One case of short circuit was reported. Emergency stop was necessary in three cases due to uncontrolled movement into the abdominal cavity. Eight of nine surgeons prefer robotic to human assistance, mostly because of a steady image and self-control.

Conclusion: The SoloAssist ${ }^{\circledR}$ robot is a reliable system for laparoscopic procedures. Emergency shutdown was necessary in only three cases. Some minor weak spots could have been identified. Most surgeons prefer robotic assistance to human assistance. We feel that the SoloAssist ${ }^{\circledR}$ makes standard laparoscopic surgery more comfortable and further development is desirable, but it cannot fully replace a human assistant.

sebastian.hollaender@uks.eu

\section{CAS.2}

\section{Development of a workflow-oriented structured report in wound} care

Martin Apitz ${ }^{1}$, Konstantin Kinzel ${ }^{1}$, Raluca Pahontu ${ }^{1}$, Beat P. MüllerStich $^{1}$, Hannes Kenngott ${ }^{1}$

${ }^{1}$ Chirurgische Klinik, Universitätsklinikum Heidelberg, Germany

Background: Postoperative wound infections are among the most common postoperative complications in general surgery. Higher incidence of wound complications and the demand for documentation and visitation are to be expected. We supposed a reduction of the length of visitation and documentation by developing a standardized report, which gives a short and structured summary of the patient history, last visitation and current therapy. Methods: The software "WoundTrack" was developed for integrating and visualizing patient data on the Google Glass ${ }^{\mathrm{TM}}$ (Google Inc., USA), using structured reporting, which reduced the patient history to relevant information. Surgical residents were enrolled in an evaluation study. They had to assess silicone wound phantoms developed from 3D scans, decide for a therapy and document them with a photograph. Patient history was developed from anonymized patient data. In terms of time and work contentment, the WoundTrack software was compared to the documentation process on a desktop PC, as it is the status quo of most surgical clinics. Results: So far, 14 surgical residents and surgeons were included. With the WoundTrack software, time to complete the requested task was significantly reduced. Grading of work contentment did not differ greatly. $71 \%$ of Google Glass ${ }^{\mathrm{TM}}$ user had enough information for a therapy decision, in comparison with $86 \%$ of desktop users.

Conclusion: Structured reporting reduced time to assess and document three postoperative wounds by reducing information load to relevant patient data. However, subjective knowledge of patient history and current therapy decreased. Further visualization technologies with Glasses have to consider this necessarily reduced provision of information.

kenni@gmx.de

\section{CAS.3}

Quantitative laparoscopy for bowel length measurement in bariatric surgery - from bench to bedside

Martin Wagner ${ }^{1}$, Benjamin Mayer $^{1}$, Sebastian Bodenstedt ${ }^{2}$, Stefanie Speidel $^{2}$, Felix Nickel ${ }^{1}$, Lars Fischer ${ }^{1}$, Beat P. Müller-Stich ${ }^{1}$, Hannes Kenngott $^{1}$

${ }^{1}$ Universitätsklinikum Heidelberg, Germany; ${ }^{2}$ Karlsruher Institut für Technologie, Karlsruhe, Germany

Background: Metabolic surgery is the recommended treatment option for patients suffering from morbid obesity. Out of the existing metabolic procedures, laparoscopic Roux-en-Y gastric bypass (LRYGB) 
is the most commonly performed. Yet it remains challenging to objectively measure the length of the bowel limbs. To address this challenge, we developed and evaluated a computer-assisted bowel length measurement system (BMS), which adds measurement functionality to conventional 3D-laparoscopes and thereby allows for quantitative laparoscopy.

Methods: BMS was used with a 3D-laparoscope (3D TipCam1, KarlStorz $\mathrm{GmbH}$, Tuttlingen, Germany). The bowel surface was reconstructed from the 3D-Image and the shortest distance between instrument tips was calculated. For thorough preclinical evaluation, phantom ( $n=50$, two users), exvivo-porcine ( $n=50$, two users) and in-vivo porcine trials ( $n=10$, five users) were performed. In all experiments a total distance of $70 \mathrm{~cm}$ was measured with BMS. Afterwards ground truth was obtained by manual measurement of the bowel, requiring laparotomy during the in-vivo porcine trials. Accuracy was calculated as ratio of ground truth to length measured with BMS. Results: Mean value measured in phantom trial was $66.1 \mathrm{~cm}$ (standard deviation (SD) $2.7 \mathrm{~cm}$ ) and accuracy of $94.43 \%$. Ex-vivo-porcine trial resulted in a mean value of $65.8 \mathrm{~cm}(\mathrm{SD} 2.55 \mathrm{~cm})$ and accuracy of $94.03 \%$. In-vivo-porcine trials a mean value of $67.5 \mathrm{~cm}$ with a standard deviation of $6.8 \mathrm{~cm}$ and an accuracy of $96.43 \%$ was achieved. Usage within a clinical setting in a human patient was feasible.

Conclusion: BMS enables safe and precise laparoscopic bowel length measurement. As application of quantitative laparoscopy it could allow for more standardized metabolic surgery.

martin.wagner@med.uni-heidelberg.de

\section{CAS.4}

\section{WatsonMD—-the IBM Watson System passes the USMLE}

Hannes Kenngott ${ }^{1}$, Martin Apitz ${ }^{1}$, Achim Rettinger ${ }^{1}$, Beat P. Müller-Stich ${ }^{1}$ ${ }^{1}$ Chirugische Klinik, Universitätsklinikum Heidelberg, Germany

Background: IBM developed the Watson system, which is capable of understanding natural language. This system is famous for competing and winning against two champions of the quiz show Jeopardy! in 2011. Since then, IBM has vastly improved the system and can be used to provide answers for questions in natural languages. We wanted IBM Watson to pass the USMLE medical state exam using an open-source knowledge base such as Wikipedia.

Methods: The medical content of Wikipedia was downloaded and processed to be the knowledge base for the Watson machine. Experts trained the machine with 100 questions of the USMLE state exam. 30 questions were randomly selected for testing. A special algorithm was developed to work with multiple choice questions.

Results: We achieved $50 \%$ of right answers with 30 questions.

Conclusion: Watson is capable to process given factual knowledge and transfer it to new questions. Therefore, the Watson system might exceed the cognitive capabilities of physicians in terms of theoretical medical knowledge. The testing questions have to be scaled up to 308 questions. To pass the USMLE the system has currently to achieve $62 \%$ of right answers.

kenni@gmx.de

\section{CAS.5}

A novel technique to perform Robotic Roux-en-Y-gastric bypass surgery: preliminary results of a matched pair

Larissa Clea Vines ${ }^{1}$, Andrea Rossetti ${ }^{1}$, Nabil Kalak ${ }^{1}$, Marc Schiesser ${ }^{1}$
${ }^{1}$ Kantonsspital St. Gallen, Switzerland

Background: Laparoscopic gastric bypass surgery is one of the most frequent procedures worldwide. Standardized operation techniques to perform robotic gastric bypass surgery are scarcely reported. We developed a novel Robot assisted Roux-en-YGastric-Bypass (R-RYGB) technique and compared it to our standard laparoscopic gastric bypass (LRYGB) technique using a matched pair analysis.

Methods: The first 20 consecutive patients undergoing R-RYGB between March and November 2015 were matched to 20 patients who underwent LRYGB. The novel R-RYGB technique adopted most of the technical features from our standard LRYGB linear stapling technique, except the gastrojejunostomy, which is performed by a hand-sewn running suture. We analyzed operation time, complication rate and length of hospital stay.

Results: The mean age was $40.4( \pm 11.3)$ years and the mean body mass index was $42( \pm 3) \mathrm{kg} / \mathrm{m}^{2}$ in both groups. The operation time was significantly longer with $138.5( \pm 27.5)$ minutes in the R-RYGB versus 87.6 $( \pm 12.3)$ minutes in the LRYGB group. Operation time dropped gradually from 162 to $107 \mathrm{~min}$ in the last patient of the R-RYGB group. There was no leakage or major complication requiring a reoperation in both groups. However, we observed 2 stenotic strictures at the gastrojejunostomy that required endoscopic dilation in the R-RYGB group. The median length of stay was similar with 5 days in the R-RYGB and 6 days in the LRYGB group.

Conclusions: Our preliminary data show that R-RYGB surgery is safe and offers similar perioperative results compared to LRYGB with a longer operation time but a steep learning curve.

larissaclea.vines@kssg.ch

\section{CAS.6}

Is robotic surgery valuable for a minimally invasive esophageal surgery program?

Matthias Biebl ${ }^{1}$, Oliver Haase ${ }^{1}$, Felix Aigner ${ }^{1}$, Sascha Chopra ${ }^{1}$, Christian Denecke $^{1}$, Andreas Brandl ${ }^{1}$, Ricardo Zorron ${ }^{1}$, Andreas Andreou ${ }^{1}$, Benjamin Strücker ${ }^{1}$, Johann Pratschke ${ }^{1}$

${ }^{1}$ Charite Universitätsmedizin Berlin, Germany

Background: Recently, minimally invasive surgery (MIS) has become adopted for tumor resection of the esophagus, however, the standard surgical technique has yet to be established. Among other techniques, the DaVinci robotic system is used for MIS of the esophagus.

Methods: After introducing a MIS program for esophageal surgery in 2014, we have adopted the DaVinci SI system for thoracic dissection of the esophagus. While the standard MIS technique comprises a 5-port laparoscopic and 4 port thoracoscopic approach, the thoracic DaVinci technique applies one camera, two robotic and two assistant ports. Anastomosis (esophagogastrostomy), if applicable, was performed using a $25 \mathrm{~mm}$ circular stapler. Decision to use the robot was either surgeon's preference, or attempted esophageal sparing resection.

Results: Of 44 consecutively operated patients, three patients $(6.8 \%$, $\mathrm{f}: \mathrm{m}=1: 2)$ underwent robotic resection. Two robotic cases were done for esophageal cancer with resection and gastric sleeve pull-up for reconstruction, one was a tumor enucleation for GIST of the lower esophagus. Docking of the robot was easy and esophageal mobilization and lymph node dissection very comfortable. However, in this limited experience, the number of harvested lymph nodes was not different from the standard MIS approach. For the case of tumor enucleation, the robot was excellent and facilitated meticulous dissection with R0 tumor removal without mucosal damage.

Conclusion: The DaVinci robot enables meticulous preparation of the thoracic esophagus with minimal trauma. It's value for routine IvorLewis resection is yet the be established.

matthias.biebl@charite.de 


\section{CRS.1}

New kid on the block: perineal stapled prolapse resection (PSP) is it worthwhile in the long-term?

Bianka Hummel ${ }^{1}$, Stephan Bischofberger ${ }^{1}$, Christine Maurus ${ }^{1}$, Walter Brunner $^{2}$, Bruno Schmied ${ }^{1}$, Lukas Marti ${ }^{1}$

${ }^{1}$ Kantonsspital St. Gallen, Switzerland; ${ }^{2}$ Spital Rorschach, Kantonsspital St. Gallen, Switzerland

Background: Perineal stapled prolapse resection (PSP) has been described as a new surgical treatment for external rectal prolapse in 2008. Short and midterm results acknowledged PSP as a safe, fast and easy procedure for high-risk patients. This study aims to assess long-term results after PSP, as such data are sparse in the literature.

Methods: All patients operated on performing a PSP from 2007 to 2015 were analysed retrospectively. Data was gathered from the medical records, operative reports, and by interviews with the general practitioner or the patient.

Results: Indication for PSP was provided for 61 patients. In one case the procedure had to be changed to an Altemeier's and in another to a laparoscopic rectopexy. The median age was 78.5 years (range 25-97), 2 patients were men. Spinal anaesthesia was used in 24 patients. The median operation time was $31 \mathrm{~min}$ (range 15-115). There was no postoperative death. Only one patient had to be reoperated, all other complications were minor. The median hospital stay was 6.5 days (range 2-23). Mean follow-up was 21.4 month (SD 21.8). Prolapse recurred in 14 patients $(22.9 \%$ ) within 9.2 month (SD10.8). Preoperative incontinence persisted in 20 patients, improved in 6 patients and terminated in 4 patients. New onset faecal incontinence was recorded in 4 cases and improved spontaneously.

Conclusion: Due to low morbidity and the possibility of spinal anaesthesia PSP is suitable for frail patients. The recurrence rate of $22.9 \%$ is similar to alternative perineal procedures like Delorme's and Altemeier's, but inferior to the laparoscopic techniques.

bianka.hummel@kssg.ch

CRS.2

Low anterior resection for rectal cancer: should the mesenteric defect be closed?

Kristjan Ukegjini ${ }^{1}$, Walter Brunner ${ }^{1}$, Rene Warschkow ${ }^{1}$, Christine Maurus ${ }^{1}$, Stephan Bischofberger ${ }^{1}$, Bruno Schmied ${ }^{1}$, Lukas Marti ${ }^{1}$.

${ }^{1}$ Kantosspital. St. Gallen, Switzerland

Background: The incidence of internal hernia after low anterior resection is unknown. The aim of this study was to analyse this problem in our patients.

Methods: All patients, in whom a rectal cancer was resected from January 2011 to December 2013, were analysed retrospectively. It was investigated, if laparoscopy, or closure of the mesenteric defect did influence the development of an internal hernia.

Results: Included were 150 patients receiving a LAR with primary anastomosis. Median follow-up was 1.2 years (range 0.01-3.0). Laparoscopic LAR was done in 53 patients (35\%) and converted in $10(7 \%)$; (primary open: $87(58 \%)$ ). The mesenteric defect was closed in 77 patients $(51 \%)$, but only in 3 laparoscopic cases. An internal hernia was seen in 4 patients $(2.7 \%)$. All of these had a laparoscopic operation without initial closure of the mesentery ( $8 \%$ hernias in this subpopulation). Laparoscopic revision was possible in two, whereas two needed open revision. In one no further problems were noted; in the others a life threatening condition developed: a necrosis of the colon, an anastomotic leakage, and a rupture of the herniated small bowel. An association of development of internal hernias with laparoscopic operation $(p<0.01)$ as well as with open mesenteric defect $(p=0.04)$ was seen. Closure of the mesenteric defect didn't cause a higher incidence of anastomotic leakage.

Conclusion: In up to $8 \%$ of laparoscopic LAR an internal hernia might develop. The problem seems to be underestimated in the literature.

kristjan.ukegjini@kssg.ch

\section{ET.3}

A new European curriculum and diploma in minimally invasive surgery

Georg Bischof ${ }^{1}$

${ }^{1}$ Evangelisches Krankenhaus, Vienna, Austria

The UEMS (www.uems.net) covers as NGO and NPO the interests and defends the professional status of more than 1.6 million doctors in Europe and has an official mandate from the European Commission in their duty. The UEMS Section and Board of Surgery (www.uemssurg.org) provide and organize the "Transferable Competencies" in our common "House of Surgery".

It is the task of the Section and Board to harmonize and standardize the highest level of specialist training and medical care respecting the EU principles of free movement of labour and services, subsidiarity and mutual recognition of qualifications and diploma. The close cooperation with scientific societies is of significant importance to achieve our common goals.

Within the Section and Board of Surgery a new Working Group "Minimal Invasive Surgery" was created in early 2015. It is the task of the proponents of this new "Transferable Competency" to elaborate a Curriculum (Definition, Syllabus, Knowledge \& Skills, LogBook) and to organize a European Board Examination and to confer an approved Board Fellowship.

In this regard we would like to propose a cooperation between the national MIS societies and the new MIS Working Group.

georgbischof@excite.com

\section{ET.5 \\ Development of a sensor- and expert model based training device for laparoscopic surgery}

Karl-Friedrich Kowalewski ${ }^{1}$, Felix Nickel ${ }^{1}$, Jonathan Hendrie ${ }^{1}$, Sebastian Bodenstedt $^{2}$, Hannes Kenngott ${ }^{1}$, Martin Wagner ${ }^{1}$, Anna-Laura Wekerle ${ }^{1}$, Stefanie Speidel ${ }^{2}$, Rüdiger Dillmann ${ }^{2}$, Beat Peter Müller-Stich ${ }^{1}$

${ }^{1}$ Klinik für Allgemein-, Viszeral- und Transplantationschirurgie, Universitätsklinikum Heidelberg, Germany; ${ }^{2}$ Karlsruher Institut für Technologie (KIT), Karlsruhe, Germany

Background: The training modalities for laparoscopic surgery are limited in their ability to provide realistic tissue interaction and objective feedback. The aim of the present study is the development of a training system that combines the advantages of training modalities such as operating on real tissue, real-time feedback and independence from trainers.

Methods: Two types of sensors were combined for motion tracking of experts and instruments. The Polaris NDI infrared camera was used for optical of the instruments. Microsoft Kinect was used for motion tracking of experts' joints while operating on a Box-Trainer. Surgical experts then performed laparoscopic tasks in a standardized fashion on a Box-Trainer; their motions were recorded. In the annotation and data analysis, each key step was assigned a specific counterpart in the tracking data. Expert models were created from this data. A virtual surgeon, the "iSurgeon," was created as a three-dimensional avatar to provide real-time feedback. 
Results: After thorough analysis we found that rotary motions are best recorded with Polaris, whereas the wrapping of the thread around the needle-holder is more accurately tracked by using the Kinect. The "iSurgeon" displayed the recorded expert models and their comparisons to the trainees' live data in a preliminary version.

Conclusion: The development of an expert model based training system for laparoscopic surgery feasibly combines the advantages of existing training modalities in order to improve learning curves and maximize training efficiency. The presented system will be compared to conventional training modalities for laparoscopic suturing and knot tying in a randomized trial.

karl.kowalewski@googlemail.com

\section{MAS.2}

Single-incision and natural orifice translumenal endoscopic surgery in Switzerland

Daniel C. Steinemann ${ }^{1}$, Andreas Zerz ${ }^{1}$, Sebastian Lamm ${ }^{1}$

${ }^{1}$ Kantonsspital Baselland, Department of Surgery, Bruderholz, Switzerland

Background: The Swiss Association of Laparo- and Thoracoscopic Surgeons (SALTS) collected procedures performed in single-incision surgery (SIS) and natural orifice translumenal endoscopic surgery (NOTES) in a prospective database. The current study aims to analyze the use of SIS and NOTES in Switzerland.

Methods: The prospective, voluntary database on surgical and endoscopic novel technologies is analyzed between the 17.08.2010 and the 24.08.2015. Results: 140 SIS and 510 NOTES procedures from 12 surgical departments were analyzed. 15 different surgeries were performed; cholecystectomy (43\%) and sigmoidectomy (26\%) were the most common procedures. Consultant surgeons performed $89 \%$ of SIS and $42 \%$ of NOTES. The proportion of teaching procedures in SIS and NOTES technique was 4 and $43 \%(p<0.0001)$. The surgeon's impression of performance was perfect in $50 \%$ of SIS and $89 \%$ of NOTES procedures $(p<0.0001)$. In NOTES technique the preferred route was transvaginal $(89 \%)$ followed by transrectal $(11 \%)$. There were $0.6 \%$ access-related complications in the NOTES and none in the SIS group. The overall morbidity was $5 \%$ in SIS and $2.7 \%$ in NOTES $(p=0.19)$. There were $3.6 \%$ of conversions in SIS and $5.7 \%$ in NOTES to conventional laparoscopy. The proportion of conversion to open surgery in SIS and NOTES together was $1.1 \%$.

Conclusion: SIS and NOTES in mainly used in routine procedures as cholecystectomy and sigmoidectomy. Surgeons using these novel techniques are highly experienced. Surgeons felt more comfortable with NOTES than with SIS and more NOTES procedures were taught to younger colleagues.

daniel.steinemann@gmx.ch

\section{MAS.3 \\ From transvaginal specimen retrieval toward hybrid NOTES sigmoid resection-results and technical evolution}

Anna Rieger ${ }^{1}$, Markus M. Heiss ${ }^{1}$, Dirk R. Bulian ${ }^{1}$

${ }^{1}$ Klinikum der Universität Witten/Herdecke, Campus Merheim, Kliniken der Stadt Köln, Klinik für Viszeral-, Gefäß- und Transplantationschirurgie, Cologne, Germany

Background: In order to reduce surgical trauma, natural orifice transluminal endoscopic surgery (NOTES) has been developed. Up to now, transvaginal cholecystectomy is the most frequently performed procedure. Here we describe our experience in 14 cases of sigmoid resection for diverticular disease and the evolution of the technique from transvaginal specimen retrieval toward transvaginal hybrid NOTES resection.

Methods: All 14 hybrid NOTES sigmoid resections performed in our clinic were analyzed regarding technique, procedural time, hospital stay, conversion rate and rate of complications.

Results: In the beginning, only transvaginal specimen retrieval was performed. One $13 \mathrm{~mm}$-, one $10 \mathrm{~mm}$ - and two $5 \mathrm{~mm}$-trocars were necessary. This technique has evolved toward a transvaginal hybrid NOTES sigmoid resection by usage of only $5 \mathrm{~mm}$-trocars and a transvaginal $13 \mathrm{~mm}$-trocar for stapling. Furthermore, the technique of anastomosis has changed over time. Over all procedures, median operation time was 192 min (139-270) with a tendency toward a reduction over time (first and second half of the procedures: 193 versus $179 \mathrm{~min}$ ). There were no conversions or intraoperative complications, but three postoperative complications were seen $(21$, $4 \%$ ). Median length of postoperative hospital stay was 7 days (728).

Conclusion: Although transvaginal retrieval of sigmoid specimen with no need of a retrieval incision is technically challenging, a step-by-step evolution toward a hybrid NOTES procedure is feasible. Our analysis found promising results in terms of procedural time, conversion- and complication-rate as well as length of hospital stay.

anna.rieger@web.de

\section{MS.1 \\ APOLLO OVERSTICH- a new procedure for Endoluminal bariatric surgery: preliminary clinical experience}

Ricardo Zorron ${ }^{1}$, Manoel Galvao ${ }^{2}$, Sasha Chopra ${ }^{1}$, Tido Junghans ${ }^{3}$, Johann Pratschke ${ }^{1}$

${ }^{1}$ Charité Universitätsmedizin Berlin, Germany; ${ }^{2}$ Gastrobeso Center, Sao Paulo Brazil; ${ }^{3}$ Klinikum Bremerhaven Reinkenheide, Germany

Background: Gastric bypass for morbid obesity can induce important excess weight loss (EWL) during 2 years after surgery, and comorbidities often improve or resolve. A weight plateau typically occurs as equilibrium in energy balance is reached after 1 year after surgery. Weight regain may be induced, among others, by anatomical factors as increased size of the anastomosis or gastric pouch, causing loss of restriction. We propose a new endoscopic procedure to reduce the gastric pouch and anastomosis, potentially reversing the typical plateau after gastric bypass and applied in a pilot clinical series.

Methods: Endoscopic revision of gastric bypass, closure of gastrocutaneous fistula, and primary endoscopic sleeve plication were performed using the full-thickness suturing device Apollo Overstich. Technical steps included: 1. Diagnostic endoscopy with measure of the pouch. 2. Insertion of the Overtube. 3. Argon burning of the whole anastomotic surface. 4. Lateral suturing of the. 5 . Sizing the GE with a $0.8 \mathrm{~mm}$ dilation balloon. The patients were followed and documented regarding complications, weight loss and co-morbidities.

Results: Patients were submitted to the procedure without intraoperative complications. Mean operative time for revisions was $126 \mathrm{~min}$. Followup showed satisfactory weight loss with no weight regain after 6 months. Conclusion: Endoscopic revision with Apollo Overstich for weight regain after gastric bypass is a new non-invasive procedure with a low learning curve and satisfactory early results. The effectivity of the use of the device for primary endoscopic sleeve plication have to be analysed in further studies.

rzorron@gmail.com 
MS.2

Weight loss, reoperations and reflux: 10 year results of laparoscopic Sleeve gastrectomy

Daniel Moritz Felsenreich ${ }^{1}$, Felix Langer ${ }^{1}$, Ronald Kefurt ${ }^{1}$, Peter Panhofer $^{1}$, Martin Schermann ${ }^{2}$, Christoph Sperker ${ }^{2}$, Philipp Beckerhinn ${ }^{3}$, Ivan Kristo ${ }^{4}$, Johannes Lenglinger ${ }^{4}$, Sebastian Schoppmann ${ }^{4}$, Gerhard Prager $^{1}$

${ }^{1}$ University Clinic for Surgery, Clinical Department for Abdominal Surgery, Medicine University Vienna, Austria; ${ }^{2}$ Hospital Rudolfsstiftung, Department for Surgery, Austria; ${ }^{3}$ Hospital Hollabrunn, Department for Surgery, Austria; ${ }^{4}$ Clinical Department of Gastrointestinal Functional Diagnostics, Medical University Vienna, Austria

Background: Beside gastric bypass, laparoscopic sleeve gastrectomy (LSG) is the most commonly performed bariatric procedure in Austria. For the long-term follow-up, the durability of the weight loss success and the incidence of clinically relevant gastro-oesophageal reflux are still under discussion.

Method: In this retrospective study, patients from three bariatric centres (Medicine University Vienna, Hospital Klosterneuburg and Hospital Rudolfsstiftung Vienna) with a follow-up of 10 or more years after LSG were included. Weight loss success, weight regain and the incidence of revision surgery was analysed as well as Quality of Life (QoL), which was surveyed by standardized questionnaires (BAROS, SF36, GIQOL, RSI, BQL). Gastro-oesophageal reflux was assessed by gastroscopy (with biopsy) as well as manometry and 24-h pH-metry.

Results: Overall, 53 patients underwent LSG until the end of 2005, at one of the three bariatric centres. The mean operative weight was $134 \pm 20 \mathrm{~kg}$, corresponding a mean BMI of $47.7 \pm 7,3 \mathrm{~kg} / \mathrm{m} 2$.

During the 10 year follow-up, a total of 19 of the 53 patients (36\%) were converted to a gastric bypass due to significant weight regain or reflux. We present in detail weight loss data and the results of the gastroscopy, manometry, 24-h pH-metry as well as data on QoL.

Conclusion: In LSG patients with a long-term follow-up of 10 years or more, a high conversion rate to a gastric bypass was observed. To make a statement on the incidence and relevance of postoperative reflux after LSG, the results of this ongoing study must be awaited.

moritz.felsenreich@meduniwien.ac.at

\section{MS.3 \\ One-anastomosis JEJ-interposition with gastric resection (Zorron-Branco Proced) for postbypass hypoglycemia}

Ricardo Zorron ${ }^{1}$, Alcides Branco ${ }^{2}$, Jose Sampaio ${ }^{2}$, Tido Junghans ${ }^{3}$ ${ }^{1}$ Charité Universitätsmedizin Berlin, Germany; ${ }^{2}$ Clinic CEVIP, Curitiba, Brazil; ${ }^{3}$ Klinikum Bremerhaven Reinkenheide, Germany

Background: The anatomic and physiologic changes of Gastric Bypass may lead to uncommon but difficult to treat complication as hyperinsulinemic hypoglycemia with neuroglycopenia. For patients non-responders for conservative treatment, extreme therapy with distal pancreatectomy or revision to normal anatomy were reported. We propose a new procedure to effectively treat this complication after bariatric surgery and applied in a pilot clinical series.

Methods: Laparoscopic revision with One-Anastomosis jejunal interposition and gastric remnant and alimentary limb jejunal resection (ZorronBranco Procedure) was performed in 4 symptomatic and irresponsive to medical treatment patients with chronic symptomatic hypoglycemia from 2 to 11 years after RYGB. Technical steps included: 1 . Fully adhesiolysis and recognition of anatomy; 2. Remnant gastrectomy with stapling ca $3 \mathrm{~cm}$ from pylorus. 3. Section of the jejunal limb $20 \mathrm{~cm}$ from GE. 4 . Handsewn anastomosis between jejunal interposition and remnant antrum. 5. Resection of the remnant alimentary limb. 6. Leak testing with methylene blue.

Results: All patients were submitted to the procedure without intraoperative complications. Mean operative time for revisions was $188 \mathrm{~min}$. Postoperative stay was 6 days. Follow-up showed normalization of insulin levels from a mean of 8.2 to $3.2 \mathrm{microUI} / \mathrm{ml}$.

Conclusion: The surgical therapy for symptomatic hypoglycemia was successful in all cases using the technique, possibly because of restoring the function of the duodenum allowing reduction of GIP and GLP-1 and hyperinsulinemia, and reducing ghrelin production due to fundic resection. Jejunal interposition is a safe therapy for hyperinsulinemic hypoglycemia post gastric bypass in selected patients.

rzorron@gmail.com

\section{MS.4}

Impact of mini gastric bypass on type 2 diabetes mellitus in comparison to sleeve gastrectomy-1 year results

Andreas Plamper ${ }^{1}$, Mareike van Lessen ${ }^{1}$, Sebastian Kolec ${ }^{1}$, Mario Musella $^{2}$, Karl Rheinwalt ${ }^{1}$

${ }^{1}$ St. Franziskus-Hospital, Germany; ${ }^{2}$ University of Neapel, Italy

Background: The role of bariatric surgery for the treatment of metabolic diseases has continuously gained importance leading to the recommendation of several international guidelines to suggest metabolic surgery as a very effective treatment option for type 2 diabetes mellitus (T2DM). Whereas bypass procedures such as biliopancreatic diversion or Roux-enY-gastric bypass have been shown to have excellent results on diabetes remission, the data on restrictive procedures such as sleeve gastrectomy (SG) is inconsistent. We compared the impact on T2DM after mini gastric bypass (MGB), a bypass procedure with increasing interest worldwide, to that after SG.

Methods: Our patients that underwent either of the 2 procedures were entered in a European multicenter study. In total, 313 obese patients with the diagnosis of T2DM were collected in the study. 175 of these patients underwent MGB and 138 patients were operated by SG. The groups were compared 1 year after the operation for BMI-loss / \%EWL, fasting plasma glucose, $\mathrm{HbAlc}$ and use of anti-diabetic medication.

Results: After the 1st postoperative year, the follow-up rate was $85.8 \%$. BMI decrease $(33.1+/-6.6$ vs. $35.9+/-5.9, p<0.001)$ as well as $\%$ EWL $(64.7+/-22.9$ vs. $52.4+/-18.3, p<0.001)$ were significantly higher in MGB. FPG and HbA1c levels were significantly lower in the MGB group. More patients were in remission for T2DM after MGB $(85.4 \%$ vs. $60.9 \%, p<0.001)$. At multivariate analysis, high baseline $\mathrm{HbA1c}$, preoperative consumption of anti-diabetic medication and T2DM duration over 10 years were negative predictors for diabetes remission while the MGB procedure represented a positive predictor.

Conclusion: Both procedures proofed to be effective for weight loss and remission of T2DM after 1 year. MGB however so far seems to have better results in comparison to SG.

andreas.plamper@cellitinnen.de

\section{V.1}

Laparoscopic distal pancreatectomy: a safe and standardized procedure

Christoph W. Strey ${ }^{1}$

${ }^{1}$ Friederikenstift Hannover, Germany

Background: Laparoscopy is currently not regarded as the standard procedure for pancreatic surgery. Distal pancreatectomy however can be 
performed safely and in a standardized manner laparoscopically. The video of a laparoscopic distal pancreatectomy demonstrates a perfect overview and technical safety of the procedure with the use of monopolar diathermy and bipolar tissue sealing.

Methods: The radiologic finding of an intraductal papillary mucinous neoplasm (IPMN), main duct type, was established in a 59 year old female patient. This led to the indication of a laparoscopic distal pancreatectomy. The patient is secured in a decubitus position with legs apart. The surgeon stands between the legs applying a four trocar technique with the assistant on the patients' right. Specimen removal is performed via a Pfannenstiel incision.

Results: Operation time was $2 \mathrm{~h} 12 \mathrm{~min}$ and no relevant blood loss was measured. Histopathology revealed a gastric main duct type IPMN with dysplasia free resection margins. No pancreatic fistula or other relevant postoperative complications were noted.

Conclusion: Laparoscopic distal pancreatectomy can be safely performed in a well defined standardized fashion.

Christoph. Strey@ddh-gruppe.de

\section{V.2}

\section{Laparoscopic enucleation of a benign pancreatic cyst-How I do it}

Christoph Michalski ${ }^{1}$, Philip C. Müller ${ }^{1}$, Beat P. Müller-Stich ${ }^{1}$, Markus Büchler ${ }^{1}$, Thilo Hackert ${ }^{1}$

${ }^{1}$ Department of General-, Visceral- and Transplant Surgery, University Hospital of Heidelberg, Germany

Background: Organ-sparing resections are indicated for benign cystic diseases of the pancreatic body or tail. The laparoscopic access offers several advantages over the open approach: less blood loss, decreased pain, a shorter hospital stay and fewer complications. We present the case of a laparoscopic cyst enucleation in the pancreatic body for a mucinous cystic neoplasia.

Methods: A 46-year old woman was administered to our clinic with upper abdominal pain. Imaging showed a $17 \times 15 \mathrm{~mm}$ cystic mass in the pancreatic corpus without communication to the pancreatic duct. CEA was slightly elevated with $3,9 \mathrm{ug} / \mathrm{l}$.

Results: The patient was placed in French-position. Three 12-mm and one 5 -mm trocar were placed. Exposure of the cystic mass at the lower dorsal border of the pancreatic corpus. The cyst was enucleated with Ultracision. Operative time was $90 \mathrm{~min}$, no intraoperative complications occurred and blood loss was minimal. The patient was discharged home after 7 days without any postoperative complications. Final pathologic diagnosis revealed a mucinous cystic neoplasia without malignancy.

Conclusion: Laparoscopic cyst enucleation of the pancreatic corpus was feasible and offers an alternative to the open approach for selected patients.

philip.mueller@med.uni-heidelberg.de

\section{V.3}

\section{Advantages and challenges in laparoscopic left lateral sectionectomy}

Moritz Schmelzle ${ }^{1}$, Daniel Seehofer ${ }^{1}$, Johann Pratschke ${ }^{1}$, Ricardo Zorron $^{1}$

${ }^{1}$ Charite - Universitätsmedizin Berlin, Germany

Background: Multi-incision laparoscopic liver resection (MILL) has become safe and is favored by most hepatobiliary centers for left lateral sectionectomy. Recent studies reported on potential advantages of single-incision approaches in extrahepatic abdominal surgery, e.g., a shorter operation time as well as improved convalescence and cosmetic results.
Methods: We here report on our first experiences with single-incision laparoscopic liver resection (SILL), exemplified in left lateral sectionectomy. Challenges and advantages are demonstrated in a sequential video comparing reduced port accesses with formal multiport laparoscopy.

Conclusion: Few experiences exist in reduced-incision liver surgery and further standardization is urgently needed. SILL appears to be technically feasible and safe and might simplify anatomical liver resection. Further technical improvements in single-ports might bear the potential to extended approaches to hilar preparation and further decrease operation times in SILL.

moritz.schmelzle@charite.de

V.8

Totally endoscopic esophagectomy—a single center experience

Martin Jazra ${ }^{1}$, Nurretin Albayrak ${ }^{1}$

${ }^{1}$ St. Anna-Hospital Herne, Germany

Background: We report our technique of the totally endoscopic esophagectomy.

Methods: Up to now, we performed this laparoscopic and thoracoscopic operation 25 times in our department. All operations have been saved on hard disk and a film was produced consisting of the important steps.

Results: None of the 25 operations had to be converted to open surgery. The oncological results proven by histological examination were equal to the open surgery. We observe benefits in terms of postoperative pain, early recovery and hospital stay. While the laparoscopic part maintained more or less the same, the development of the thoracoscopic part was a learning process. Patient's position, trocar placement and anastomotic technique have been repeatedly optimized. During thoracoscopy the patient is located in a $45^{\circ}$ left lateral position, a mini-thoracotomy serves to take out the specimen and introduce the OrVil stapler. The oral part of the stapler is protected by an additional purse-string suture we perform endoscopically. We use a silicone drain fixed by a suture to transfer the stomach to the right pleural cavity, a high intrathoracic end-to-side anastomosis is established.

Conclusion: In our opinion, the high intrathoracic anastomosis represents the most challenging part of the minimally invasive esophagectomy. Our current approach has proven to be a safe and practical technique of the totally endoscopic Ivor-Lewis procedure.

martin.jazra@elisabethgruppe.de

P.2

Interactive and immersive three-dimensional surgery planning of complex liver resection

Anas Preukschas ${ }^{1}$, Arianeb Mehrabi ${ }^{1}$, Stefanie Speidel ${ }^{2}$, Micha Pfeiffer ${ }^{2}$, Matthias Huber ${ }^{2}$, Lisa Bettscheider ${ }^{1}$, Beat P. Müller-Stich ${ }^{1}$, Hannes Kenngott ${ }^{1}$

${ }^{1}$ Chirurgische Universitätsklinik Heidelberg, Heidelberg, Germany: ${ }^{2}$ Karlsruher Institut für Technologie, Karlsruhe, Germany

Background: Surgical resection is mostly the definite treatment of liver lesions. Despite recent advances, morbidity rates remain high. Operation planning promises to achieve oncologically safe resection margins and ensure maximum residual liver tissue. We present a new, interactive and immersive method of visualizing preoperative planning data with a 3D virtual reality framework using a head-mounted display (HMD).

Methods: A sample case of a patient who underwent liver resection was selected. We segmented the liver, gallbladder, vasculature, bile ducts and the liver tumors from computed tomography images. We used the HMD 
to visualize the intraoperative anatomical situation in the abdomen. By using the HMD the surgeon could access the $3 \mathrm{D}$ visualized upper abdomen, clinical patient data and the original CT images. We evaluated user satisfaction, acceptance and potential by an online questionnaire.

Results: We evaluated the system with attending surgeons $(n=13)$, resident surgeons $(n=34)$, medical students $(n=57)$, surgical nurses $(n=52)$ and non-medical staff $(n=22)$. In total 180 evaluations were performed. $89 \%$ of the users were satisfied with the virtual reality scene, $90 \%$ saw its potential to better evaluate complex surgical cases, $87 \%$ found it useful in the training of medical students, $85 \%$ in surgical training and $56 \%$ in the training of nurses. Eighty percent saw high clinical potential of this technology.

Conclusion: 3D surgery planning and simulation in combination with immersive virtual reality can prove beneficial for complex liver resections. It may also help younger surgeons to better understand the underlying anatomy of a surgical case and the reasoning behind the surgical decision-making processes.

kenni@gmx.de

\section{P.5}

Satisfaction, ODS score, incontinence and recurrence after laparoscopic ventral rectopexy

Sara Notz ${ }^{1}$, Stephan Bischofberger ${ }^{1}$, Walter Brunner ${ }^{1}$, Bruno Schmied ${ }^{1}$, Lukas Marti ${ }^{1}$

${ }^{1}$ Kantonsspital St. Gallen, Switzerland

Background: Laparoscopic ventral rectopexy (LVR) is a relatively new procedure for the treatment of complete rectal prolapse as well as obstructed defecation syndrome (ODS). The aim of this study was to assess the functional outcome, as well as recurrence rate and satisfaction after LVR.

Methods: All 73 female patients operated on with a LVR from December 2010 to April 2014 were enrolled. Complete rectal prolapse was the cause for operation in 32 patients. In 41 patients complex pelvic floor prolapse causing ODS was the reason to operate. All patients were reviewed in the outpatient clinic 6 weeks and 6 months postoperatively. Anatomical recurrence, satisfaction, preoperative and postoperative fecal incontinence as well as obstructive defecation symptoms were assessed.

Results: LVR was performed successfully in all patients, although four needed laparotomy. Median age was 68 years (range 49-87). Reoperation was necessary (once caused by a tacker irritating a lumbal nerve, twice an early ileus occurred, furthermore a dehiscence in the vagina was found and a superficial wound dehiscence emerged) in five (7\%), all recovered completely. One rectal prolapse recurred (3\%). Fecal incontinence improved remarkably. ODS symptoms were reduced in 35 of 41 patients. Median satisfaction was 9 (range 2-10) on a scale from 0 to 10 .

Conclusion: LVR is an effective technique for the correction of rectal prolapses and ODS. It may be the procedure of choice for complete rectal prolapse as it leads to postoperative improvement of constipation and incontinence. Therefore, satisfaction is high and makes some risk of complications acceptable.

sara.notz@kssg.ch

\section{P.6}

Feasibility of hybrid-NOTES cholecystectomy in morbidly obese patients-a comparison to normal weight patients

Anna Rieger ${ }^{1}$, Jürgen Knuth ${ }^{2}$, Markus M. Heiss ${ }^{1}$, Dirk R. Bulian ${ }^{1}$ ${ }^{1}$ Klinikum der Universität Witten/Herdecke, Campus Merheim, Kliniken der Stadt Köln, Klinik für Viszeral-, Gefäß- und Transplantationschirurgie, Cologne, Germany; ${ }^{2}$ Klinikum Kempten-
Oberallgäu, Klinik für Allgemein-, Viszeral-, Gefäß- und Thoraxchirurgie, Kempten, Germany

Background: Many advantages for Transvaginal Hybrid-NOTESCholecystectomy (TVC) have been described comparing this technique to traditional laparoscopic cholecystectomy. Obesity is considered as an exclusion criterion in many studies although it is a well-known risk factor for cholecystolithiasis. Therefore, a separate evaluation of this patientgroup is necessary.

Methods: Out of all 197 TVC performed in our clinic, the 16 morbidly obese patients (BMI $\geq 40.0 \mathrm{~kg} / \mathrm{m} 2$; MOP) were compared to the 59 normal weight patients (BMI 18.5-24.9 kg/m2; NWP) concerning the rate of complications and conversions as well as patient- and procedure-related parameters.

Results: Median operation time was significantly longer for MOP as compared to NWP (76.5 min [41-159] vs. 51.0 min [31-140]; $p=0.001)$. For MOP, significantly more often the application of more than one percutaneous trocar was necessary $(43.8 \%$ vs. $5.1 \%$; $p<0.001)$. In one case, closure of the transvaginal access was impossible due to obesity. There were no conversions or intraoperative complications, and 4 postoperative complications only in the NWP group $(6.8 \%)$, but without a significant difference between the two groups $(p=0.572)$. No significant difference in the length of postoperative hospital stay could be seen ( 2 days [2-6] vs. 2 days [1-7]; $p=0.814$ ).

Conclusion: Although TVC technically seems to be more challenging in morbidly obese patients, our analysis could find no disadvantage in terms of conversion- or complication-rate and length of hospital stay.

anna.rieger@web.de

P.7

Splenic torsion with repeated splenic infarction-laparoscopic splenectomy for a rare pathology

Anna Rieger ${ }^{1}$, Markus M. Heiss ${ }^{1}$, Dirk R. Bulian ${ }^{1}$

${ }^{1}$ Klinikum der Universität Witten/Herdecke, Campus Merheim, Kliniken der Stadt Köln, Klinik für Viszeral-, Gefäß- und Transplantationschirurgie, Cologne, Germany

Background: An uncommon complication of wandering spleen is splenic torsion leading to splenic infarction. The most accepted theory for etiology of wandering spleen - which is a diagnostic challenge - is laxity of the splenic ligaments. Only few cases of wandering spleens remaining intermittently in orthotopic position were reported.

Methods: Case report

Results: The case of an 18-year-old female patient who presented with recurrent left upper quadrant pain is reported. A CT scan revealed splenomegaly, partial splenic infarction and portosystemic collateral vessels. There was no reason for these symptoms found preoperatively, especially no signs for a splenic torsion due to its orthotopic position and even screening for thrombophilia could not find any irregularities. Laparoscopic splenectomy was performed without any complication after angiographic coiling of the splenic artery. Anatomy first remained unclear intraoperatively with a spleen allegedly in orthotopic position and "normal" rotation. A $360^{\circ}$-torsion around the vascular pedicle could be seen with a consecutive venous stasis and collateral vessels when adhesiolysis had been performed. Splenic detorsion and splenectomy could be done subsequently. The patient could be discharged from hospital on the sixth postoperative day after an uncomplicated postoperative course. Conclusion: Splenic torsion is a diagnostic challenge, especially if the spleen is in normal position. The definitive diagnosis in our case could only be made intraoperatively. A possible surgical treatment is detorsion 
and splenopexy, but if infarction already has happened, splenectomy has to be performed, and this should be done preferably in a laparoscopic manner.

anna.rieger@web.de

\section{P.8}

Laparoscopic treatment of Dunbar syndrome

Petr Skala ${ }^{1}$

${ }^{1}$ Kreiskrankenhaus Rotenburg an der Fulda, Germany

Background: Dunbar is rare disorder defined as postprandial abdominal pain, weight loss and an abdominal bruit related to compression of the celiac artery by median arcuate ligament.

Methods: case report.

Results: A 27-year old male patient with chronic abdominal pain in epigastrium, dyspepsia and weight loss that have been ongoing on for over 2 years. Clinical and laboratory examinations were with normal findings, esophagoduodenoscopy with low grade gastritis, abdominal sonography also without pathology. Duplex ultrasonography showed flow turbulence in celiac trunk with peak systolic velocities $191 \mathrm{~cm} / \mathrm{s}$. MRIAngiography showed stenosis of the celiac trunk by median arcuate ligament. After adequate preoperative assessment, transection of the median arcuate ligament was performed. Postoperative course was without complications and the patient was discharged 8th postoperative day. Postoperative controls shows improvement of symptoms and increase of weight. Conclusion: Preoperative selection and surgical procedure may significantly reduce illness symptoms in these patients. Classic treatment for Dunbar syndrome is represented by open surgery with celiac artery decompression. Laparoscopic procedure as a safe and reliable technique may increase benefits of patients. However additional trials are necessary to confirm the validity of this procedure.

p.skala@kkh-rotenburg.de

\section{P.11}

Comparison of methods for bowel length measurement in bariatric surgery: results of a phantom trial

Benjamin Mayer ${ }^{1}$, Martin Wagner ${ }^{1}$, Sebastian Bodenstedt ${ }^{2}$, Stefanie Speidel $^{2}$, Beat P. Müller-Stich ${ }^{1}$, Hannes G. Kenngott ${ }^{1}$

${ }^{1}$ Klinik für Allgemein-, Viszeral- und Transplantationschirurgie, Universitätsklinikum Heidelberg, Germany; 2: Institut für Anthropomatik und Robotik, Karlsruher Institut für Technologie, Karlsruhe, Germany

Background: Laparoscopic Roux-en-Y gastric bypass (LRYGB) surgery is the most commonly performed bariatric procedure. The length of the constructed bowel limbs has an effect on success of treatment. However only $53 \%$ of bariatric surgeons measure the lengths of bowel limbs. Therefore a phantom trial was performed to evaluate accuracy, traumatic impact and usability of bowel measurement methods compared to a novel computer-assisted bowel measurement system developed in our group (BMS).

Methods: Four bowel length measurement methods were compared in a phantom trial: guessing, distance marks on laparoscopic graspers, premeasured umbilical tape and BMS. Trials were performed with a 3D-laparoscope (3D TipCam 1 Spies $^{\mathrm{TM}}$, Karl Storz GmbH) on bowel phantoms within a standard box trainer. A total of 7 measurements until $70 \mathrm{~cm}$ were conducted. Start and end point of each measurement were marked with pins and total distance was measured manually. Also time and number of bowel grasps were recorded.
Results: Preliminary results showed that participants measured bowel most accurate with umbilical tape $(68.9+/-2.95 \mathrm{~cm})$ and BMS $(68.65$ $+/-1.85 \mathrm{~cm})$. Bowel had to be grasped most often when applying laparoscopic distance markers $(14.7+/-3.83)$ and least with guessing $(14.7+$ + $-3.83)$ and BMS (14.71. +/- 2.94).

Conclusion: Bowel length measurement methods commonly used by most bariatric surgeons are not accurate and account for more bowel grasps, suggesting a higher potential for bowel trauma than other methods. Further experiments with more participants are necessary to validate the preliminary results.

benjamin.mayer@med.uni-heidelberg.de

\section{P.13 \\ Intragastric single port surgery (IGS) for large benign gastric tumors: clinical series}

Ricardo Zorron ${ }^{1}$, Sasha Chopra ${ }^{1}$, Tido Junghans ${ }^{2}$, Johann Pratschke ${ }^{1}$ ${ }^{1}$ Charité Universitätsmedizin Berlin, Germany; ${ }^{2}$ Klinikum Bremerhaven Reinkenheide, Germany

Background: Local gastric resection is usually indicated in benign gastric tumors with a diameter beyond $3 \mathrm{~cm}$ if full-thickness resection is needed or endoscopic removal is impossible. As an alternative we present a new technique of intragastric single port surgery (IGS) for the resection of solitary large benign tumors of the stomach.

Methods: Five patients with benign tumors localized at submucosa level with a diameter range between 3-6 cm encompassing GISTs, Dieulafoy angiodysplasia and adenomas were included. As a next step the stomach wall is percutaneous exteriorized and a single port was introduced in the epigastric space under direct vision. The resection was performed with $60 \mathrm{~mm}$ linear staplers und endoscopic guidance and the tumor was retrieved through the single port access. Hand-sutured oversewing was routinely performed and the gastric incision was closed.

Results: The operation time lasted between 42 and $58 \mathrm{~min}$ for the intragastric surgery. No intraoperative complications were observed. One patient developed a wound infection. All resection margins were tumor free. Postoperative control at POD 15 revealed no complications. Conclusion: The concept of percutaneous intragastric single port gastric surgery (IGS) facilitates the minimal invasive resection of large benign gastric tumors with reduced loss of unaffected gastric tissue.

rzorron@gmail.com

\section{P.16}

Single-incision laparoscopic surgery for diverticulitis

Henning Spieker ${ }^{1}$

${ }^{1}$ University Hospital Leipzig, Department for Visceral, Transplantation, Thoracic and Vascular Surgery, Leipzig, Germany

Background: Single-incision laparoscopic surgery (SILS) is frequently used for indications such as appendectomy, cholecystectomy or sigmoid surgery. An umbilical incision is used as primary access and as well for specimen retrieval. Nevertheless, an umbilical access for SILS is may be associated with technical limitations in the pelvic area. We used a Pfannenstiel incision for SILS sigma or anterior rectum resection for complicated diverticulitis.

Methods: SILS sigma or anterior resection was performed using a Pfannenstiel access to the abdominal cavity. A Gelport with three trocars was used. In one patient, an elective resection after initially conservative treatment for a covered perforation was performed, two patients underwent resection due to an abscess and fistula, and in two patients 
an early resection was performed due to abdominal abscesses. One Patient received an appendectomy and a suture of the bladder after a complicated appendicitis. All patients suffered from sigmoid diverticulitis, which was stage IIb according to Hansen and Stock.

Results: The operation time ranged from 89 to $280 \mathrm{~min}$. There were no conversions, and no additional trocars were used. The postoperative hospital stay ranged from 5 to 14 days. All patients were discharged without any intraoperative or postoperative complications.

Conclusions: SILS sigma or anterior rectum resection for complicated diverticulitis can be performed via a Pfannenstiel incision. This approach provides direct visualization and access into the pelvis as well as the option to benefit from open surgery devices. The Pfannenstiel incision may generally be recommended for the favorable cosmetic effect and the very low rate of incisional hernias.

Henning.Spieker@medizin.uni-leipzig.de

P.17

\section{Percutaneous laparoscopy: new Teleflex equipment—first} experiences

Christine Wurst ${ }^{1}$, Matthias C. Raggi ${ }^{1}$

${ }^{1}$ Agaplesion Bethesda Hospital, Department of Surgery, Stuttgart, Germany

Background: Using the new Percuvance system from Teleflex it's possible to perform laparoscopic surgery percutaneous using a reduced number of trocars, with less than $3 \mathrm{~mm}$ incisions. Experience was limited to the initial generation. A minimal learning curve was noted.

Methods: Within this first generation it was possible to use several instruments. There is a reusable handle combined with a single use shaft and single use tooltip. It is possible to attach different $5 \mathrm{~mm}$ tooltips at the end of the shaft. To bring the instrument into the abdomen a blunt tip introducer is used. Up to now groin hernia, cholecystectomy, fundoplication and bariatric procedures were performed.

Results: The Percuvance shaft must firstly be attached to the handle. The various tooltips must also be attached to the shaft. It is necessary to engage a lock button on the first generation Percuvance system. With the Introducer it is easy to get the instrument into the abdominal cavity through a $1-\mathrm{mm}$ incision. We used the $5 \mathrm{~mm}$ camera trocar to exchange the Percuvance introducer for a grasper tooltip. The shaft has the required stiffness while providing some flexibility that allows for angulation without too much of a bend. All mentioned surgeries could be performed in a safe manner without any need of conversion to conventional instruments. Conclusion: At present important tooltips are not yet available A standard $5 \mathrm{~mm}$ trocar is required. While using only one camera trocar, desufflation of smoke is problematic. In terms of cosmetics and safety very good results are provided.

christine.wurst@bethesda-stuttgart.de

\section{P.21}

Preventing staple line leaks after sleeve gastrectomy by fluorescence enhanced laparoscopy

Clara Böker $^{1}$, Julian Mall ${ }^{1}$

${ }^{1}$ KRH Klinikum Nordstadt, Hannover, Germany

Background: Laparoscopic sleeve gastrectomy is progressively winning recognition. By now the sleeve gastrectomy is the leading bariatric procedure in Germany. Reduction of obesity related comorbidities and weight loss as well as complication rates are comparable to the Roux en Y-gastric bypass. Staple line leakage with an incidence up to $5 \%$ is a well-known complication that can present with intraabdominal abscess, sepsis or multiorgan failure. $90 \%$ of the leaks occur at the gastroesophageal junction close to the angle of His. Besides mechanical factors local ischemia is discussed to be responsible for staple line leaks.

Methods: Therefore we are investigating fluorescence enhanced laparoscopy with indocyanine green to estimate staple line perfusion in 100 sleeve gastrectomies. Fluorescence imaging will be assessed qualitatively as well as quantitatively. The aim of this study is the identification of possibly poor perfused regions of the sleeve.

Conclusion: Whether the intraoperative measurements may have immediate implications on the surgical treatment in those identified patients, e.g., endoscopic stenting has yet to be defined.

clara.boeker@gmx.de 


\section{Index of authors}

Aigner F. CAS.6

Albayrak N. V.8

Andreou A. CAS.6

Apitz M. CAS.2, CAS. 4

Beckershinn P. MS.2

Belfontali V. 3D.2, 3D.4

Bellemann N. 3D.1

Bettscheider L. P.2

Biebl M. CAS.6

Birk D. CAS. 1

Bischof G. ET.3

Bischofberger S. CRS.1, CRS.2, P.5

Bodenstedt S. CAS.3, ET.5, P.11

Böker A. P.21

Branco A. MS.3

Brandl A. CAS.6

Brunner W. CRS.1, CRS.2, P.5

Büchler M. W. 3D.1, V.2

Bulian D. R. MAS.3, P.6, P.7

Chopra S. CAS.6, MS.1, P.13

Denecke C. CAS.6

Dillmann R. ET.5

Fedtke K.

Felsenreich D. M. MS.2

Fischer L. CAS.3
Galvao M. MS.1

Gondan M. 3D.1

Guigard S. 3D.2, 3D.4; 3D.5

Haase O. CAS.6

Hackert T. V.2

Heiss M. MAS.3, P.6, P.7

Hendrie J. ET.5

Holländer S. CAS.1

Huber M. P.2

Hummel B. CRS.1

Jazra M. V.8

Junghans T. MS.1, MS.3, P.13

Kalak N. CAS. 5

Karenovics W. 3D.2, 3D.4; 3D.5

Kauczor H. U. 3D.1

Kefur R. MS.2

Kenngott H. G. 3D.1, CAS.2, CAS.3, CAS.4, ET.5, P.2, P.11

Knuth J. P.6

Koch A. BD. 4

Köckerling F. BD.2

Kolec S. MS.5

Kowalewski K.-F. ET.5

Kristo I. MS.2

Lamm S. H. MAS.2

Langer F. MS.2

Lenglinger J. MS.2

Licker M. 3D.5
Mall J. P.21

Marti L. CRS.1, CRS.2, P.5

Maurus C. CRS.1, CRS.2

Mayer B. CAS.3, P.11

Mehrabi A. P.2

Meinzer H. P. 3D.1

Michalski C. V.2

Müller-Stich B. P. 3D.1, CAS.2, CAS.3, CAS.4, ET.5, V.2, P.2, P.11

Müller M. 3D.1

Müller P. C. V.2

Musella M. MS.5

Nickel F. 3D.1, CAS.3, ET.5

Notz S. P.5

Panhofer P. MS.2

Pfeiffer M. P.2

Plamper A. MS.5

Prager G. MS.2

Pratschke J. CAS.6, MS.1, V.3,

P.13

Preukschas A. 3D.1, P.2

Radeleff B. 3D.1

Raggi M. C. P.17

Rheinwaldt K. P. MS.5

Rieger A. MAS.3, P.6, P.7

Rossetti A. CAS.5

Sadowski S. 3D.2, 3D.4

Sampaio J. MS.3
Schermann M. MS.2

Schiesser M. CAS.5

Schmelzle M. V.3

Schmied B. CRS.1, CRS.2, P.5

Schön M. R.

Schoppmann S. MS.2

Seehofer D. V.3

Simon T. BD.2

Skala P. P.8

Speidel S. CAS.3, ET.5, P.2, P.11

Sperker C. MS.2

Spieker H. P.16

Steinemann D. C. MAS.2

Stock C. 3D.1

Strey C. W. V.1

Strücker B. CAS.6

Triponez F. 3D.2, 3D.4; 3D.5

Ukegjini K. CRS.2

Van Lessen M. MS.5

Vidal Fortuny J. 3D.2, 3D.4, 3D.5

Vines L. C. CAS.5

Wagner M. 3D.1, CAS.3, ET.5, P.11

Wekerle A. L. 3D.1, ET.5

Wurst C. P.17

Zerz A. MAS.2

Zorron R. CAS.6, MS.1, MS.3, V.3, P.13 